\title{
Two-mirror aplanatic telescopes with a flat field
}

\author{
V. Yu. Terebizh*† \\ Crimean Astrophysical Observatory, \\ Nauchny, Crimea 298409, Ukraine
}

February 10, 2022

\begin{abstract}
A complete description is given of two-mirror telescopes with a flat medial focal surface, on which the images of stars are circles of least confusion. Particular attention is paid to aplanats, since their field of view is noticeably larger than that of classical systems. Two sets of appropriate solutions correspond to Schwarzschild and Gregorian telescopes. As a result, it becomes possible to use flat light detectors with wide-field two-mirror telescopes. New designs are of particular interest when as few reflective surfaces as possible are required, which is typical for space exploration and non-optical observations.
\end{abstract}

\section{Introduction}

By definition, aplanatic optical systems are those in which spherical aberration and coma are corrected. For a pure mirror aplanat, the field of view is limited primarily by astigmatism, curvature of the focal surface, and, to a lesser extent, by distortion. The combined action of astigmatism and field curvature results in two focal surfaces of paraboloidal shape, tangential and sagittal, with the paraxial radii of curvature $R_{t}$ and $R_{s}$, on which the images of

*E-mail: valery@terebizh.ru

${ }^{\dagger}$ Experimental Astronomy DOI: 10.1007/s10686-022-09833-0 
stars are elongated in mutually perpendicular directions (see, e.g., Hecht [1], p. 264). Generally speaking, further correction of astigmatism does not ensure a flat focal surface: the tangential and sagittal surfaces merge with each other, forming a single Petzval surface with the finite paraxial radius of curvature $R_{P}$, and only the addition of the Petzval condition provides a flat shape of the focal surface (Born and Wolf [2], Section 5.5.3; Korsch [3], Section 6.5).

The complete definition of the two-mirror system contains too few initial parameters, namely two, to correct astigmatism and field curvature, so the main instruments of the 20th century were two-mirror aplanats, which include the Schwarzschild, Ritchey-Chrétien systems and Gregory-Maksutov mirror aplanats (Schroeder [4], Terebizh [5]). In particular, the Hubble Space Telescope (HST) is a Ritchey-Chrétien aplanat with radii of curvature $R_{t}=-574.730 \mathrm{~mm}, R_{s}=-693.941 \mathrm{~mm}$, and $R_{P}=-774.237 \mathrm{~mm}$. All image surfaces here are concave with respect to the direction of the light rays, and the Petzval surface has the smallest curvature.

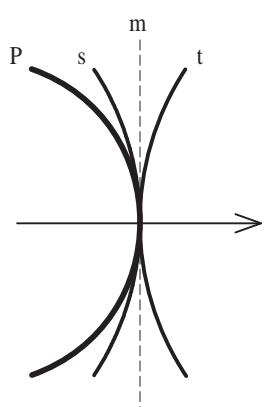

a

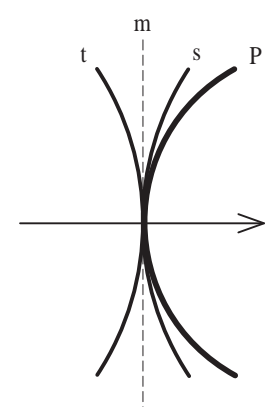

b

Figure 1: Two types of image surfaces allowing a flat medial surface. P, s, m and t denotes the Petzval, sagittal, medial, and tangential focal surfaces, respectively.

Since flat light detectors still prevail, two-mirror telescopes with flat image surfaces, be they classical systems or aplanats, are of undoubted interest. Some special cases of aplanatic two-mirror telescopes with a flat field are known (for example, Schwarzschild exact aplanat), but this problem has not been systematically considered. Such a study can be carried out with the help of the procedure called artificial flattening (Hecht [1], p. 269). It consists in the arrangement of the tangential and sagittal surfaces symmetrically relative to the focal plane, and then the medial surface lying between them will be flat 
(Figure 1). Of course, images of stars on the medial surface (circles of least confusion) are still spoiled by astigmatism, but they are the smallest and round. Pay attention: it is desirable to make flat not the notional Petzval surface, but precisely the medial surface.

The purpose of this paper is to give a simple and complete description of two-mirror telescopes that combine the wide field of view of aplanats with the possibility of using flat radiation detectors.

\section{Arbitrary two-mirror telescope}

Let us recall two results obtained by Joseph Petzval in the middle of the 19th century (Born and Wolf [2]). The first is the relation

$$
\frac{3}{R_{s}}-\frac{1}{R_{t}}=\frac{2}{R_{P}}
$$

that is valid for an arbitrary optical system. 1 Besides, the tangential and sagittal surfaces are always on the same side of the Petzval surface. The second result is a special case of the general representation of the Petzval radius for a two-mirror system:

$$
\frac{1}{R_{P}}=-\frac{2}{R_{1}}+\frac{2}{R_{2}},
$$

where $R_{1}$ and $R_{2}$ are paraxial radii of curvature of the primary and secondary mirrors, respectively.

In general, the medial surface is specified by the condition

$$
\frac{1}{R_{m}} \equiv \frac{1}{2}\left(\frac{1}{R_{t}}+\frac{1}{R_{s}}\right),
$$

which requires its curvature to be the arithmetic mean of the curvature of the tangential and sagittal surfaces.

For what follows, it is necessary to find explicit representations of all four radii of curvature in terms of the parameters of the optical system.

Various sets of initial parameters are used to describe two-mirror telescopes; for both the optical systems and their aberrations we use the simple

\footnotetext{
${ }^{1}$ Equation (1) is in the wrong place in the original version of the Experimental Astronomy article.
} 


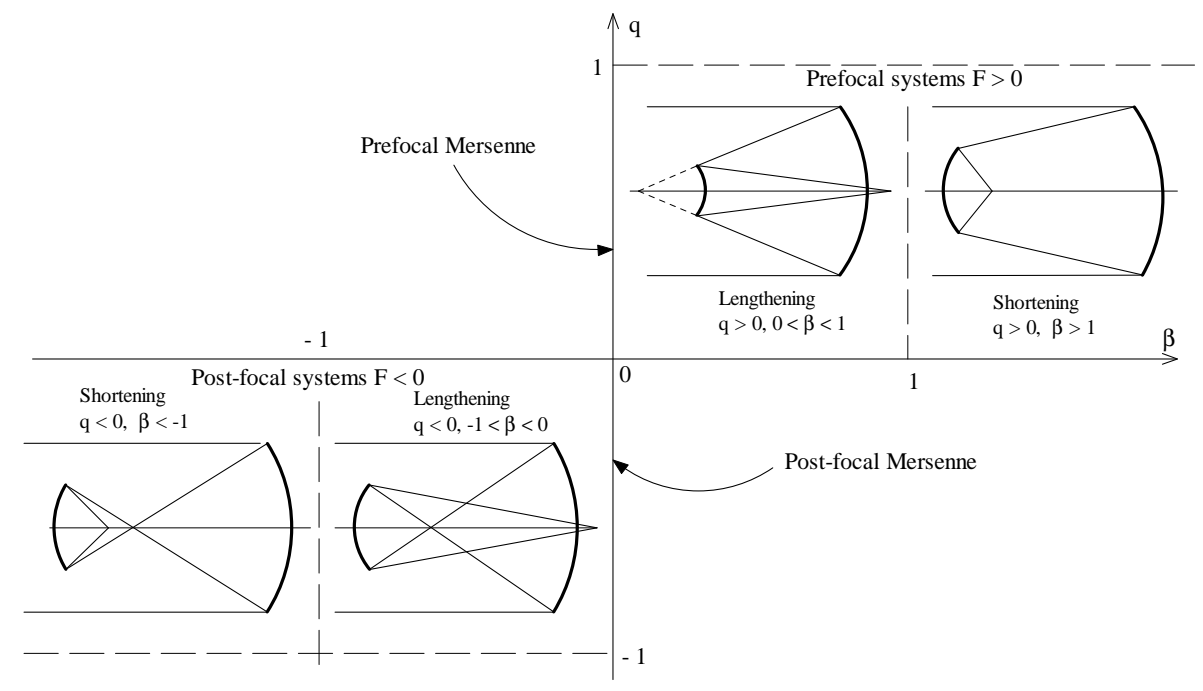

Figure 2: Schematic representation of two-mirror telescopes on the Maksutov diagram. First and third quadrants correspond to systems in which secondary mirror is located, respectively, before the primary focus and behind it; two other quadrants correspond to systems with virtual images. Further subdivision of systems is determined by whether the focal length of the primary mirror is lengthened or shortened by the secondary mirror. Mersenne systems correspond to the $\beta=0$ axis.

description by Maksutov [6,7]. Extension of this approach to arbitrary twomirror telescopes is illustrated in Figure 2 (Terebizh [5]). The scheme comes from four parameters: the telescope aperture diameter $D$, the equivalent focal length $F$, and two dimensionless ratios

$$
q=s_{2}^{\prime} / F, \quad \text { and } \beta=f_{1} / F,
$$

where $s_{2}^{\prime}>0$ is the back focal length of the telescope, that is the distance from the secondary mirror to the axial focal point, and $f_{1}$ is the focal length of the primary mirror. As usually, we assume $f_{1}=-R_{1} / 2>0$ for a concave primary mirror. The value of $F$ can be either positive or negative 2 Let $D_{2}^{(0)}$

\footnotetext{
${ }^{2}$ Recall that the effective focal length $F$ of an optical system is measured from the back principal plane to the focal plane. Therefore, the negativity of $F$, as is the case for the Gregorian system, means that the back focal plane is located farther behind the focus along the optical axis. To facilitate the use of various sources, we give the relationship between the Schroeder [4] and Maksutov [6,7] variables: $m_{S c h r}=1 / \beta=m, \beta_{S c h r}=q(1+1 / \beta)-1$.
} 
be the secondary mirror light diameter for the axial incident light beam; then $|q|=D_{2}^{(0)} / D \leq 1$ is the linear obstruction ratio for the above beam, whereas $\beta$ is the inverse magnification $m=F / f_{1}$ of the secondary mirror. Parameters $q$ and $\beta$ have the same sign for telescopes with real images. As Figure 2 shows, we have positive $F$ for the Cassegrain and Schwarzschild systems, and negative $F$ for the Gregorian telescopes.

In Maksutov variables, $R_{1}=-2 \beta F$, and $R_{2}=q R_{1} /(1-\beta)$, so equation (2) gives the Petzval radius

$$
R_{P}=-\frac{q \beta F}{1-q-\beta}
$$

This expression is true for all two-mirror telescopes, whereas the form of $R_{t}$ and $R_{s}$ depends on the specific type of system.

\section{Two-mirror aplanat}

When considering aplanatic two-mirror telescopes, we proceeded from the general theory of third-order aberrations, as expressed by Maksutov [6] and Mikhelson [8]. Rather cumbersome calculations, which we omit here, lead to the following expressions for the paraxial radii of curvature of the image surfaces, namely, the tangential surface:

$$
R_{t}=-\frac{2 q \beta F}{4 \beta-(1-q)\left(3 \beta^{2}-2\right)},
$$

and the sagittal surface:

$$
R_{s}=-\frac{2 q \beta F}{(1-q)\left(2-\beta^{2}\right)} .
$$

It is easy to check that the last three formulas agree with the general Petzval relation (1). Further, it follows from equations (3), (6) and (7) that

$$
R_{m}=-\frac{q \beta F}{\beta+(1-q)\left(1-\beta^{2}\right)}
$$

for aplanatic telescopes. Referring again to the HST example with its effective focal length $F=57599.869 \mathrm{~mm}$ and parameters $q=0.111219$, and $\beta=0.095834$, we get $R_{m}=-628.735 \mathrm{~mm}$ from equation (8). 


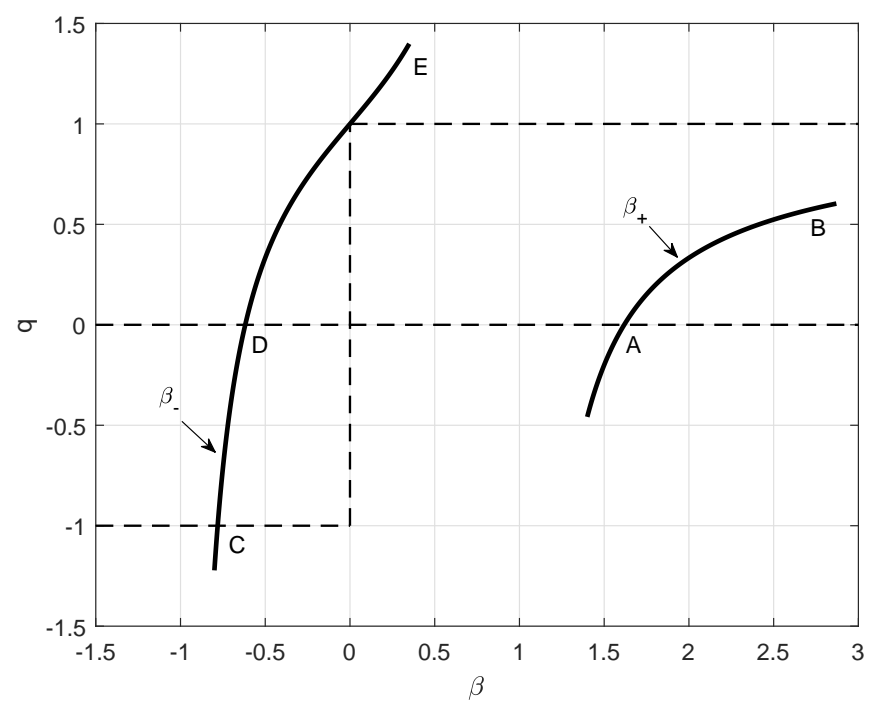

Figure 3: Bold lines in the Maskutov diagram correspond to the solutions $\beta_{+}$ and $\beta_{-}$with a flat medial surface. Only the $A B$ branch and the $C D$ section are in the zones of physically acceptable optical systems, bounded by the dotted lines.

As stated in the Introduction, by requiring $R_{t}=-R_{s}$ we arrive at one of the symmetrical arrangements of the tangential and sagittal surfaces relative to the medial surface, shown in Figure 1. The same result follows from (8) by equating the denominator to zero. The corresponding aplanatic systems with a flat medial surface are defined in the plane $(q, \beta)$ by equation

$$
q=1+\frac{\beta}{1-\beta^{2}},
$$

or its solutions

$$
\beta_{ \pm}(q)=\frac{1 \pm \sqrt{1+4(1-q)^{2}}}{2(1-q)}
$$

in the form of a quadratic equation with respect to $\beta$ (Figure 3). Comparison of Figures 2 and 3 shows that the first of these solutions corresponds to the shortening Schwarzschild aplanat in the interval $\beta \geq(1+\sqrt{5}) / 2 \simeq$ 1.618 (branch $\mathrm{AB}$ in Figure 3), whereas the second one - to the lengthening Gregory-Maksutov aplanat in the interval $(1-\sqrt{17}) / 4 \leq \beta \leq(1-\sqrt{5}) / 2$, that is approximately $-0.781 \leq \beta \leq-0.618$ (section CD in Figure 3). 
Note that both branches $\mathrm{AB}$ and $\mathrm{CD}$ imply $R_{2}>0$, i.e. concave shape of the secondary mirror. Since $R_{1}<0$ for the primary mirror, it follows from (2) that $R_{P}>0$ for the two-mirror aplanats with a flat medial surface. Thus, the curvatures of both branches are represented by the option 'b' in Figure 1.
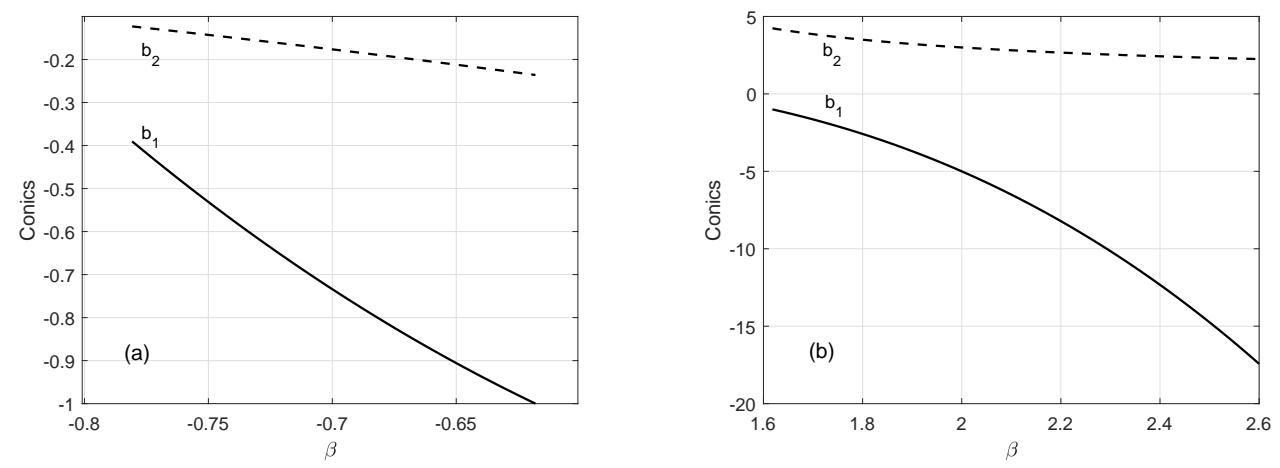

Figure 4: Conic constants for the two-mirror telescopes with a flat medial surface. Figure (a) corresponds to systems with the negative effective focal length $F$, Figure (b) - to systems with positive $F$.

The above results provide a complete description of the sought systems, but it is useful to add that relations (9) and (10) reduce the two-parameter representation of telescopes in the plane $(q, \beta)$ to a one-parameter representation of flat-field aplanats, either by means of $q$ or by means of $\beta$. Equations (2.6) - (2.13) of Terebizh [5] concerning the first-order characteristics of two-mirror systems are simplified accordingly. If we choose $\beta$ as the initial parameter, then the obscuration coefficient $q$ is given by equation (9), the back focal length $s_{2}^{\prime}=q F$, the radius of curvature of the primary mirror $R_{1}=-2 \beta F$, the same parameter for the secondary mirror

$$
R_{2}=-\frac{2 \beta F}{1-\beta}\left(1+\frac{\beta}{1-\beta^{2}}\right)
$$

and the spacing between mirrors

$$
T=\frac{\beta^{2} F}{1-\beta^{2}} .
$$


As for the conic constants of mirrors, equations (2.16) of the mentioned book take the form for flat-field aplanats:

$$
b_{1}=-1+2 \beta\left(1+\beta-\beta^{2}\right), \quad b_{2}=-\frac{1+\beta}{1-\beta} .
$$

Last equations are presented graphically in Figure 4. We see that at negative values of $F$, both mirrors have the shape of prolate ellipsoids of revolution. This was to be expected, since the mirrors of all aplanats in the region $(q<0 ;-1<\beta<0)$ have this shape. At $F>0$, that is, in the area of the Schwarzschild aplanats, the primary mirror is a hyperboloid, while the secondary is an oblate ellipsoid.

Table 1: Two designs with different signs of $F$.

\begin{tabular}{clll}
\hline Parameter & Design A & Design B \\
\hline$D$ & 1000.0 & 1000.0 \\
$F$ & 3000.0 & -3000.0 \\
$\beta$ & 2.00 & -0.70 \\
- & & & \\
$q$ & $1 / 3$ & & -0.372549 \\
$R_{1}$ & -12000.0 & -4200.0 \\
$R_{2}$ & 4000.0 & 920.415225 \\
$T$ & -4000.0 & -2882.352941 \\
$s_{2}^{\prime}$ & 1000.0 & 1117.647059 \\
$b_{1}$ & -5.0 & -0.734000 \\
$b_{2}$ & 3.0 & -0.176471 \\
$R_{t}$ & -3000.0 & -754.966887 \\
$R_{s}$ & 3000.0 & 754.966887 \\
$R_{P}$ & 1500.0 & 377.483444 \\
\hline
\end{tabular}

Note Linear dimensions are given in millimeters. The input parameters are separated by a line. $T$ means the distance between the mirrors, $s_{2}^{\prime}$ - the back focal length. 


\section{Examples of aplanatic designs}

Let us consider two examples corresponding to branches $\beta_{+}(q)$ and $\beta_{-}(q)$ of solutions with a flat medial surface.

For the Design A, we took the aperture diameter $D=1.0 \mathrm{~m}$, the effective focal length $F=3.0 \mathrm{~m}$, and $\beta=2.0$, which, according to (9), corresponds to $q=1 / 3$. These data are sufficient to calculate all the other parameters of the telescope; they are shown in the second column of Table 1. Entering numerical values into the ZEMAX program shows that the images of point sources are round throughout the entire flat field of view of the order of 20 arc minutes in diameter.

Similar data for the Design B are shown in the third column of Table 1. In this case, we adopted the same aperture, but the negative effective focal length $F=-3.0 \mathrm{~m}$, and $\beta=-0.70$. Again, the images of the stars are round in the flat field of view with a diameter greater than $1^{\circ}$. Another thing is that the images at the edges of the field become blurred, but their symmetrical shape indicates the flatness of the focal surface.

\section{Classical telescopes}

Systems with a flat medial surface exist also among classical two-mirror telescopes, that is, telescopes in which only spherical aberration is corrected. We pay less attention to these systems, since coma noticeably limits their field of view.

Within the framework of the theory of third-order aberrations, the radii of curvature of the tangential and sagittal image surfaces are now:

$$
\begin{gathered}
R_{t}=-\frac{q \beta F}{2 \beta+(1-q)\left(1-3 \beta^{2}\right)} \\
R_{s}=-\frac{q \beta F}{(1-q)\left(1-\beta^{2}\right)} .
\end{gathered}
$$

Taking into account (3), we obtain the radius of curvature of the medial surface:

$$
R_{m}=-\frac{q \beta F}{\beta+(1-q)\left(1-2 \beta^{2}\right)} .
$$

The Petzval radius $R_{P}$ is still given by (5). 
Like before, symmetrizing the arrangement of the tangential and sagittal surfaces in the form $R_{t}=-R_{s}$ or zeroing the denominator in (16) leads to the condition of flatness of the medial surface:

$$
q=1+\frac{\beta}{1-2 \beta^{2}},
$$

and its solutions

$$
\beta_{ \pm}(q)=\frac{1 \pm \sqrt{1+8(1-q)^{2}}}{4(1-q)}
$$

as a quadratic equation with respect to $\beta$. This time there are also two branches of physical solutions: the first in the interval $\beta>1$ within the first quadrant of the $(q, \beta)$ plane, and the second branch in the interval $-1 / \sqrt{2}<\beta<-1 / 2$ within the third quadrant of this plane (see Figure 2). Otherwise, the results are similar to those for aplanatic systems.

\section{Concluding remarks}

The above analytical calculations and numerical examples discuss the options for the existence of two-mirror telescopes with round images of stars on a flat detector of light within the field of view typical of aplanats. Unfortunately, the set of these systems is not abundant. The reason lies in the small number of free parameters that describe two-mirror telescopes. In this respect, greater opportunities provide three-mirror telescopes, for which it is possible to correct in addition both astigmatism and field curvature, and thereby achieve a true anastigmatic system. Corresponding algorithms for calculating three-mirror anastigmats were proposed by Korsch [3] and Terebizh $[5,9]$.

Despite the relative scarcity of flat-field two-mirror models, it is likely that they may be of interest for astrophysical and astrometric studies when as few reflective surfaces as possible are required, which is typical for space exploration and off-optical observations.

\section{Acknowledgments}

I am grateful to the anonymous Referee for helpful comments. 


\section{References}

[1] Hecht, E.: Optics, 3rd Ed. Addison-Wesley, Reading (1998).

[2] Born M., Wolf E.: Principles of Optics, 7th Ed. Cambridge Univ. Press (1999).

[3] Korsch, D.: Reflective Optics. Academic Press, Boston (1991).

[4] Schroeder, D.: Astronomical Optics. Academic Press, San Diego (2000).

[5] Terebizh, V.: Survey Telescope Optics. SPIE Press, Bellingham (2019).

[6] Maksutov, D.: Anaberration reflecting surfaces and systems, and new ways to test them. Trans. of the State Opt. Inst. (GOI), 86, 1-119 (1932).

[7] Maksutov, D.: Astronomical Optics (in Russian). Gostechizdat (1946).

[8] Mikhelson, N.: Optical Telescopes, Theory and Framework (in Russian). Moscow, Nauka (1976).

[9] Terebizh, V.: Algorithm for calculating anastigmatic three-mirror telescopes. Experim. Astronomy, 49, 85-95 (2020). 